\title{
The biology of low grade MALT lymphoma
}

\author{
P G Isaacson, J Spencer
}

In the early 1980s Isaacson and Wright described a group of extranodal low grade B cell lymphomas that recapitulated the histological features of mucosa associated lymphoid tissue (MALT) rather than those of lymph nodes. ${ }^{12}$ MALT lymphomas arise in a wide variety of anatomical sites of which the stomach is the commonest; gastric MALT lymphomas have therefore been characterised in the greatest detail. MALT lymphomas from different sites are linked not only by their histological features but also by certain aspects of their pathogenesis, clinical behaviour, and molecular genetic properties.

Clinically, low grade MALT lymphomas are distinguished by their quite remarkable indolence. ${ }^{3}$ This group of lymphomas tends to remain localised to their site of origin for extremely long periods. Although spread to regional lymph nodes often occurs, distant dissemination to the bone marrow and other tissues is only infrequently present at the time of diagnosis. This is in direct contrast to low grade B cell lymphomas of lymph nodes which characteristically disseminate to the marrow and other lymphoid tissues early in their course. Explanations for the favourable clinical course of low grade MALT lymphomas have included suggestions that they are not truly malignant but are, instead, "pseudolymphomas", that their ability to disseminate is limited by lymphocyte homing phenomena, and that their growth is influenced by exposure to antigen.

There is good evidence that MALT lymphomas are malignant neoplasms. As judged by both immunohistochemical and molecular techniques they are clearly monoclonal. ${ }^{4}$ They have also been shown to display a characteristic clonal cytogenetic abnormality in the form of trisomy 3 which can be detected in over $50 \%$ of cases. ${ }^{5}$ MALT lymphomas are invasive; they commonly erode gastric mucosa and are capable of invasion through the gastric wall to the peritoneum. Dissemination to regional lymph nodes is common, and more distant spread of MALT lymphomas to the bone marrow and other organs is well documented. ${ }^{6}$ Moreover, it has been shown that the primary and disseminated tumour are representative of the same neoplastic clone. ${ }^{6}$

It has been known for many years that large dividing $\mathbf{B}$ cells which leave activated gut associated lymphoid tissue and enter the blood are able to home back to the gut, where they differentiate into plasma cells. ${ }^{7}$ More recently it has been shown that subsets of circulating lymphocytes express homing receptors which bind to site specific endothelial ligands, the so called "vascular addressins". ${ }^{8}$ This system is thought to mediate the preferential recirculation of lymphocyte subsets through specific anatomical sites and has been considered as a possible explanation for the tendency of low grade MALT lymphomas to remain localised. There is some evidence that such mechanisms may influence the behaviour of low grade MALT lymphomas. In immunoproliferative small intestinal disease (IPSID), ${ }^{9}$ which is a subtype of gastrointestinal MALT lymphoma, the lymphoma usually arises in the proximal jejunum and shows marked plasma cell differentiation. The neoplastic plasma cells are characteristically found in mesenteric lymph nodes and throughout the intestinal lamina propria in the absence of evidence of spread to peripheral lymphoid tissue, including the bone marrow. The distribution of these plasma cells could be considered to be a reflection of the homing proclivities of normal MALT. It is possible that site specific trafficking of lymphocytes could also explain the multifocal nature of gastric MALT lymphoma, ${ }^{10}$ the frequent occurrence of bilateral salivary gland ${ }^{11}$ and conjunctival lymphoma, ${ }^{12}$ and the tendency for MALT lymphomas to involve other extranodal mucosal sites, as exemplified by gastric lymphomas spreading to the intestine and conjunctival lymphomas to the lung. However, there is no evidence as yet to support the existence of such a finely tuned receptor-ligand system which could mediate the movement of lymphocytes into single or small combinations of mucosal sites.

It is possible that the pattern of growth of low grade MALT lymphomas, as outlined above, is due to dependence on help provided by a local immune response to a specific antigen. In the case of gastric lymphoma, for example, an immune response to a local antigen in the gastric mucosa and gastric lymph nodes may promote growth of neoplastic B cells, whereas cells reaching peripheral sites fail to grow in the absence of antigenic stimuli. This could also account for such phenomena as the multifocality of gastric lymphoma and bilaterality of conjunctival lymphoma. This possibility is supported by an experiment in which an antiidiotype was raised against the tumour immunoglobulin in a case of gastric lymphoma. ${ }^{13}$ In this case a scattered population of cells 
expressing the idiotype, presumably tumour cells, was identified in the marginal zone of the spleen in the absence of any evidence for the growth of lymphoma in that organ when assessed by conventional criteria.

Certain histological features of low grade MALT lymphoma suggest that its growth is subject to immunological drive. Transformed blast cells are always found scattered in the lymphomatous infiltrate, and plasma cell differentiation is a consistent feature. In gastrointestinal MALT lymphomas, plasma cell differentiation is usually maximal beneath the surface epithelium, consistent with the presence of an antigen either in the epithelium or the lumen. Follicular colonisation, ${ }^{14}$ another distinctive property of low grade MALT lymphoma, also appears to be an antigen related phenomenon. The large number of $T$ cells that typify low grade MALT lymphomas is consistent with immunological activity within the tumours. More objective evidence for the role of antigen in MALT lymphoma is provided by the effect of broad spectrum antibiotics in IPSID. ${ }^{15}$ In a proportion of IPSID cases administration of these antibiotics has caused regression of the lymphoma. Presumably this effect is due to eradication of a bacterial antigen from the small intestine.

Paradoxically, MALT lymphomas only infrequently arise from sites where MALT is normally present, such as the tonsil and Peyer's patches. Most arise in organs, like the stomach, which normally do not contain lymphoid tissue. The explanation for this seems to be that the lymphomas arise in lymphoid tissue that has been acquired as a result of some pre-existing disorder. This is best illustrated by MALT lymphomas of the salivary gland ${ }^{11}$ and thyroid ${ }^{16}$ which arise against a background of myoepithelial sialadenitis and Hashimoto's thyroiditis respectively. The lymphoid tissue that accumulates in these two conditions has the features of MALT, being characterised by B cell follicles and an adjacent lymphoepithelium (that is, intraepithelial B cells). In the stomach the presence of lymphoid tissue with MALT characteristics is virtually pathognomonic of Helicobacter pylori infection ${ }^{17}$ and this observation has led to studies investigating the relationship of $H$ pylori to gastric MALT lymphoma. $H$ pylori can be identified in over $90 \%$ of cases of gastric MALT lymphoma, suggesting a direct link between the infection and lymphoma. ${ }^{18}$ This link has been substantiated by epidemiological studies ${ }^{19}$ and a case-control study ${ }^{20}$ which, importantly, also showed that the infection precedes the onset of lymphoma.

The close association of $H$ pylori with gastric MALT lymphoma has led to consideration that this organism might provide the antigenic stimulus that appears to be critical for the growth of the lymphoma. Studies aimed at defining the immunological specificity of the neoplastic B cells were, however, disappointing in this respect. The tumour-derived immunoglobulin failed to recognise $H$ pylori antigens but instead recognised a variety of tissue auto-antigens. ${ }^{21}$ However, in a series of carefully controlled experiments, cells teased from resection speci- mens of low grade gastric MALT lyphoma were cocultured with 13 clinical strains of heat killed $H$ pylori. ${ }^{22}$ In each of three cases a single and different strain of the organism caused the lymphoma cells to proliferate and synthesise tumour specific immunoglobulin. The organisms also induced IL2 receptor (CD25) expression by the tumour cells and secretion of IL 2, presumably by T cells, into the culture medium. Removal of tumour associated $T$ cells from the culture system abrogated all responses. Subsequent experiments (unpublished) have shown that the proliferation of the neoplastic $B$ cells requires contact dependent help from $H$ pylori specific T cells.

In a parallel clinical study ${ }^{23}$ six patients with biopsy proven low grade gastric MALT lymphoma, in all of whom $H$ pylori had been identified, were treated with appropriate antibiotics to eradicate the organism. In five of these patients the lymphoma regressed and was no longer diagnosable by histological or molecular genetic means. As more patients have been treated it has emerged that in some cases, although eradication of $H$ pylori has resulted in clinical and histological remission, molecular genetic evidence of the neoplastic clone may persist. ${ }^{24}$ While eradication of $H$ pylori is clearly an appropriate measure for all cases of gastric MALT lymphoma, it is not yet clear whether clinical remissions will be confined only to early stage (that is, mucosal) low grade disease or if cases of more advanced stage or of high grade histology will respond.

It is likely that the growth of low grade MALT lymphomas of sites other than the stomach is also dependent on locally activated $T$ cells. The challenge here is to identify the appropriate antigens and carry out the relevant therapeutic trials. A much broader question is whether the growth of nodal low grade B cell lymphomas is similarly promoted by intratumoral $\mathrm{T}$ cells despite the ease with which they disseminate. The histology and immunohistology of follicular lymphoma, mantle cell lymphoma, and lymphocytic lymphoma provide abundant tantalising evidence that these tumours, too, are immunologically active. The identification of immunological mechanisms supporting the growth of these currently incurable neoplasms would clearly be of major clinical significance.

1 Isaacson PG, Wright DH. Malignant lymphoma of mucosaassociated lymphoid tissue. A distinctive type of B-cell lymphoma. Cancer 1983;52:1410-6.

2 Isaacson PG, Wright DH. Extranodal malignant lymphoma arising from mucosa-associated lymphoid tissue. Cancer 1984;53:2515-24.

3 Cogliatti SB, Schmid U, Schumacher U, Eckert F, Hansmann ML, Hedderich J, Takahashi $\mathrm{H}$, et al. Primary B-cell gastric lymphoma: a clinicopathological study of 145 patients. Gastroenterology 1991;101:1159-70.

4 Spencer J, Diss TC, Isaacson PG. Primary B cell gastric lymphoma: a genotypic analysis. Am $\mathcal{F}$ Pathol 1989;135: 557-64.

5 Wotherspoon AC, Finn T, Isaacson PG. Numerical abnormalities of chromosomes 3 and 7 in lymphomas of mucosa associated lymphoid tissue and the splenic marmucosa associated lymphoid tissue

ginal zone. Lab Invest 1994;70:124.
Diss TC, Peng HZ, Wotherspoon AC, Pan LX, Speight $P M$, Isaacson PG. A single neoplastic clone in sequential biopsies from a patient with primary gastric MALT lymphoma and Sjogren's syndrome. N Engl f Med 1993;329: $172-5$.

7 Hall JG, Parry D, Smith M. The distribution and detection of lymph bone immunoblasts after intravenous transfer into syngeneic recipients. Cell Tissue Kinet 1992;5:269-72. 8 Butcher EC, Scollay RG, Weissman IL. Organ specificity 
of lymphocyte migration: mediation by highly selective lymphocyte interaction with organ specific determinants on high endothelial venules. Eur $\mathscr{f}$ Immunol 1980;10: 556-61.

9 Khojasteh A, Haghshenass $M$, Haghighi P. Immunoproliferative small intestinal disease. A third world lesion. $N$ Engl $₹$ Med 1983;308:1401-5.

10 Wotherspoon AC, Doglioni C, Isaacson PG. Low-grade gastric B-cell lymphoma of mucosa-associated lymphoid tissue (MALT): a multifocal disease. Histopathology 1992 20:29-34.

11 Hyjek E, Smith WJ, Isaacson PG. Primary B cell lymphoma of salivary gland and its relationship to myoepithelial sialadenitis. Hum Pathol 1988;19:766-76.

12 Wotherspoon AC, Diss TC, Pan LX, Schmid C, Kerr-Muir MC, Hardman-Lea S, et al. Primary low-grade B-cell lymphoma of the conjunctiva: a mucosa-associated lymphoid tissue type lymphoma. Histopathology 1993;23:417-24.

13 Spencer J, Diss TC, Isaacson PG. A study of the properties of low-grade mucosal B-cell lymphoma using a monoclonal antibody specific for the tumour immunoglobulin. $\mathcal{F}$ Pathol 1990;160:231-8.

14 Isaacson PG, Wotherspoon AC, Diss T, Pan L. Follicular colonization in B-cell lymphoma of MALT. Am $f$ Surg Pathol 1991;15:819-28.

15 Ben-Ayed F, Halphen $\dot{M}$, Najjar T, et al. Treatment of alpha chain disease-results of a prospective study in 21 Tunisian patients by the Tunisian-French intestinal lymphoma study group. Cancer 1989;63:1251-6.

16 Hyjek E, Isaacson PG. Primary B cell lymphoma of the thyroid and its relationship to Hashimoto's thyroiditis. Hum Pathol 1988;19:1315-26.

17 Genta RM, Hamner HW, Graham DY. Gastric lymphoid follicles in Helicobacter pylori infection: frequency, distribution, and response to triple therapy. Hum Pathol 1993; 24:577-83.

18 Wotherspoon AC, Ortiz-Hidalgo C, Falzon MR, Isaacson PG. Helicobacter pylori-associated gastritis and primary B-cell gastric lymphoma. Lancet 1991;338:1175-6.

19 Doglioni C, Wotherspoon AC, Moschini A, de Boni M, Isaacson PG. High incidence of primary gastric lymphoma in northeastern Italy. Lancet 1992;339:834-5.

20 Parsonnet J, Hansen S, Rodriguez L, et al. Helicobacter pylori infection and gastric lymphoma. $N$ Engl $f$ Med pylori infection and

21 Hussell T, Isaacson PG, Crabtree JE, Dogan A, Spencer J. Immunoglobulin specificity of low grade B-cell gastrointestinal lymphoma. Am F Pathol 1993;142:285-92.

22 Hussell T, Isaacson PG, Crabtree JE, Spencer J. The response of cells from low-grade B-cell gastric lymphomas of mucosa-associated lymphoid tissue to Helicobacter pylori. Lancet 1993;342:571-4.

23 Wotherspoon AC, Doglioni C, Diss TC, Pan LX, Moschini A, de Boni M, et al. Regression of primary low-grade Bcell gastric lymphoma of mucosa-associated lymphoid cell gastric lymphoma of mucosa-associated lymphoid tissue type after eradication of Helicobacter pylori. Lancet
1993;342:575-7.

24 Wotherspoon AC, Doglioni C, de Boni M, Spencer J, Isaacson PG. Antibiotic treatment for low-grade gastri MALT lymphoma [letter]. Lancet 1994;343:1503. 